\title{
Self-Noise of the MET Angular Motion Seismic Sensors
}

\author{
Egor V. Egorov, Ivan V. Egorov, and Vadim M. Agafonov \\ Center for Molecular Electronics, Moscow Institute of Physics and Technology, 9 Institutskiy Pereulok, Dolgoprudny, \\ Moscow 141700, Russia \\ Correspondence should be addressed to Vadim M. Agafonov; agvadim@yandex.ru
}

Received 29 October 2014; Revised 5 January 2015; Accepted 5 January 2015

Academic Editor: Yanbiao Liao

Copyright ( $\odot 2015$ Egor V. Egorov et al. This is an open access article distributed under the Creative Commons Attribution License, which permits unrestricted use, distribution, and reproduction in any medium, provided the original work is properly cited.

\begin{abstract}
Interest to angular motion seismic sensors is generated by an expectation that direct measurement of the rotations, associated with seismic signals, would allow obtaining more detailed and accurate information from them. Due to the seismic signals low intensity a self-noise of the sensors is one of the most crucial parameters, characterizing their performance. In seismic applications the molecular-electronic transfer (MET) technology is considered as one of the most promising technologies for the rotations measurements. In this research we have developed a noise model for the MET angular sensors. The experimental part of the research which fully agrees with theoretical data includes the instrument self-noise measurement in quite locations. Based on the modelling we have revealed the directions of further research to improve the MET angular sensors performance.
\end{abstract}

\section{Introduction}

Unlike traditional geophones angular motion seismic sensors are not sensitive to translational vertical or horizontal motions and generate an output signal only in presence of ground or structure rotations. Interest to angular motion seismic sensors is stimulated by an expectation that direct measurements of the rotations, associated with seismic signals, would allow estimating more precisely the response of the structures to seismic input, providing more accurate measurement of the seismic field spatial distribution, and separating modes of seismic waves based on their polarization and determine site effect [1-3].

For seismic applications, the angular motion sensors should be capable of a better than $0.1 \mu \mathrm{rad} / \mathrm{sec}$ resolution and display low sensitivity to linear motion. Taking into account that the angular seismic sensors could be largely used in oil and gas seismic exploration, their compactness, low cost, and low power consumption are to be the essential requirements. Nowadays, among a variety of technologies, the molecularelectronic transfer (MET) technology is, likely, the only one to offer a reasonably priced commercial product of the required performance [4]. The sensors based on this technology are also known as electrochemical angular motion sensors. Although the MET sensors appeared to be useful for many applications, some of them require significantly better performance than that one currently achieved.

In this paper, we concentrate our efforts on the analysis of the MET angular sensors self-noise. Improvement of the noise characteristics for MET angular sensors is not possible without understanding of the physical mechanisms, responsible for the self-noise generation. We have experimentally investigated the MET angular sensor self-noise for the range $1-150 \mathrm{~Hz}$, which covers the frequencies most significant for seismic exploration. Additional experiments and analysis of the possible noise sources allowed defining the processes responsible for the sensor self-noise at different frequencies. The noise model has been developed and compared with the experimental data. Finally, the methods for the self-noise improvement are suggested.

\section{Materials and Methods}

2.1. Instruments. The critical part of the angular motion sensors is a transducer. The mechanical configuration of the angular motion transducer, based on the MET technology, is presented in Figure 1. The transducer consists of a toroidal channel, filled with a highly concentrated iodide-iodine water-based electrolyte. An expansion volume allows compensating temperature expansions of the liquid. The sensitive 


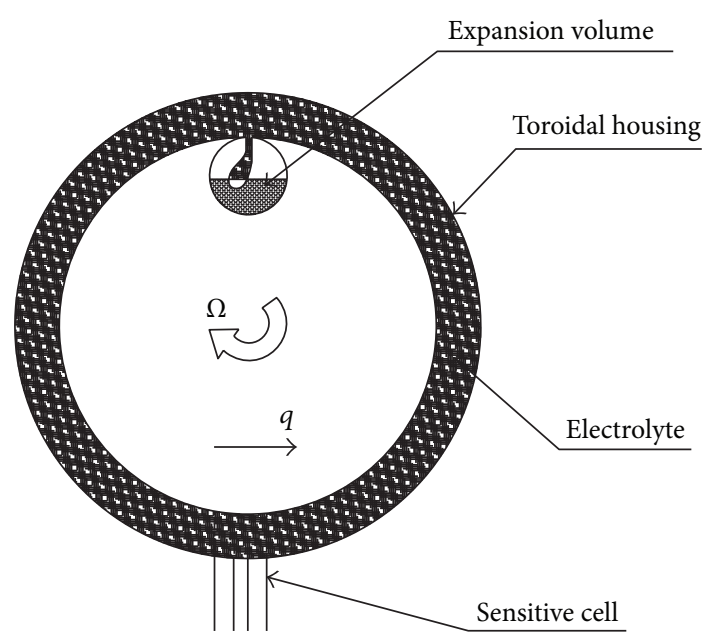

FIGURE 1: The MET angular sensor mechanical configuration.

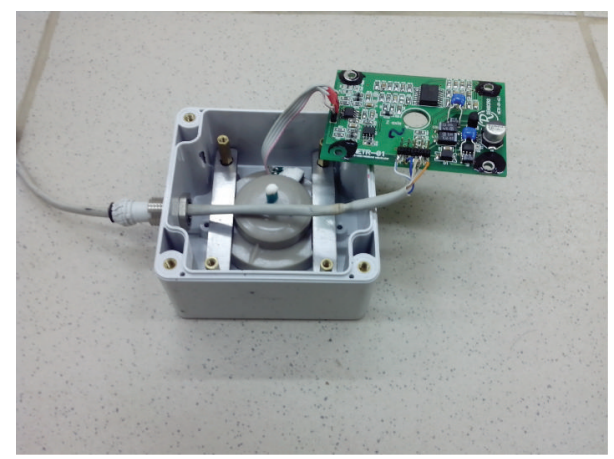

FIGURE 2: View of the angular seismic sensor used in the experiments.

cell placed across the channel converts liquid motion inside the channel into the electrical response. For the commercial sensors the sensitive cell consists of four mesh electrodes sandwiched together with three porous dielectric ceramic spacers [5]. Alternative configurations for sensitive cells have been reported recently [6-9]. External electrodes (anodes) are connected to positive potential relative to the internal ones (cathodes). The operating principles are based on the sensitivity of the active ions distribution and currents passing through the electrodes to the electrolyte motion. Commonly the differential cathodic current is used as an output signal of the MET transducer. The electrodes of the cell are connected to the signal conditioning electronic board, which converts the differential output current from the cell into voltage and shapes the response in specified frequency operational range.

In our experiments we used the METR-11 $(1-150 \mathrm{~Hz}$ frequency operational range) sensor manufactured by $\mathrm{R}$ sensors, LLC (http://www.r-sensors.ru/). The instrument used in the tests comprises the ceramic transducer with the electrodes inside, filled with an electrolyte and electronic board. The parts are held together by an external case (see photo on Figure 2). The manufacturer specified that scale factor is $K=50 \mathrm{~V} / \mathrm{rad} / \mathrm{sec}$.
The transducer toroid has external diameter of $50 \mathrm{~mm}$, and the squared cross-section of the toroidal channel is $6 \times$ $6 \mathrm{~mm}$ in size. The electrodes are made from platinum mesh with cells of $170 \times 170 \mu \mathrm{m}$ and wire diameter of $45 \mu \mathrm{m}$. The dielectric spacers are of $\sim 120 \mu \mathrm{m}$ thick with 80 round through-holes $300 \mu \mathrm{m}$ in diameter each.

The block diagram of the electronic signal conditioning board is shown in Figure 3. The first stage (marked as "stage 1" in Figure 3) is designated for transducer output current transformation to voltage and for scale factor temperature compensation. The second stage ("stage 2") is responsible for high frequency correction and for additional frequencydependent temperature compensation. The third stage ("stage 3 ") contains low pass and high pass second-order Butterworth filters having the cut-off frequencies $1 \mathrm{~Hz}$ and $150 \mathrm{~Hz}$, correspondingly.

For further analysis we need to know the instrument transfer function, which could be presented by a product of the MET transducer transfer function $W_{\text {trans }}$ with transfer functions of three stages of the electronic circuitry $W_{1 s t}, W_{2 \text { nd }}$ and, $W_{3 \mathrm{rd}}$ :

$$
W_{\text {inst }}=W_{\text {trans }} W_{1 \mathrm{st}} W_{2 \mathrm{nd}} W_{3 \mathrm{rd}} .
$$

The MET transducer transfer function $W_{\text {trans }}$ converts rotation rate $\omega$ into the differential cathodic current $I_{\text {diff: }}$ :

$$
I_{\text {diff }}=W_{\text {trans }} \omega .
$$

According to the data given in [10], $W_{\text {trans }}$ behaves differently depending on frequency range. It grows up $\sim f$ from 0 to approximately $\sim 0.1 \mathrm{~Hz}$, and then it does not depend on frequency up to $\sim 10 \mathrm{~Hz}$ and goes down to $\sim 1 / f$ at higher ones.

The electronics transfer functions could be calculated analytically or modeled using electronic designer's standard software. The first stage converts differential cathodic current $I_{\text {diff }}$ into the first-stage output voltage:

$$
U_{1}=W_{1 \mathrm{st}} I_{\mathrm{diff}}=R_{\mathrm{fb}} I_{\text {diff }} .
$$

Here $R_{\mathrm{fb}}$ is an equivalent resistance in the feedback of the first-stage operational amplifier. In practice, as it could be seen on Figure $3, R_{\mathrm{fb}}$ is made of several temperature dependent and permanent resistors, connected in sequence and in parallel, thus providing compensation of the transducer sensitivity temperature variations.

Transfer functions of the second and third stages are shaped to achieve flat response $\left|W_{\text {inst }}\right| \approx K=50 \mathrm{~V} / \mathrm{rad} / \mathrm{sec}$ of the instrument in the operating frequency range with low and high cut-off frequencies at 1 and $150 \mathrm{~Hz}$, correspondingly. The calculated frequency behavior of the product $\left|W_{2 n d} W_{3 r d}\right|$ is shown in Figure 4.

2.2. Experiments and Data Analysis. In the first set of our experiments we measured the instrument self-noise. For that purpose we placed two METR-11 sensors in the basement on the solid concrete foundation with sensitivity axis directed vertically upward. The recording was made by 24 -bit digitizer LTR-24 (http://www.lcard.ru/) over the quietest nighttime 


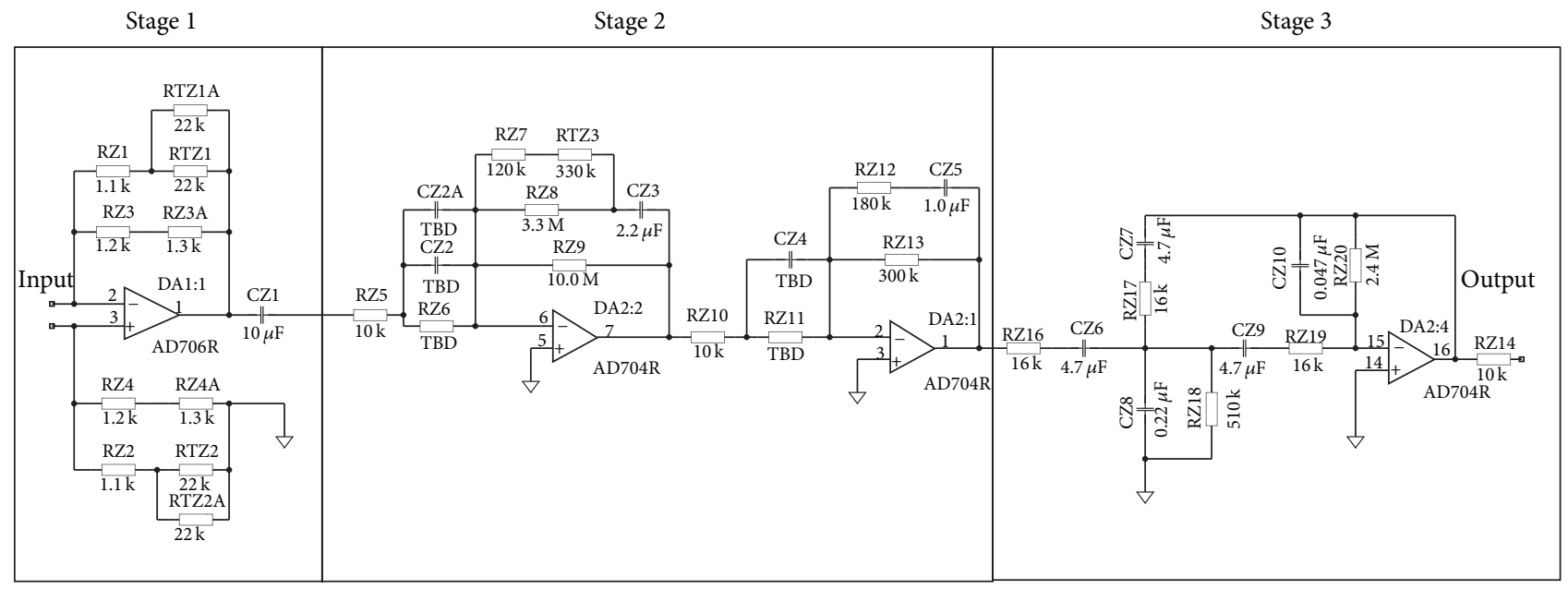

FIGURE 3: Block diagram of the signal conditioning board.

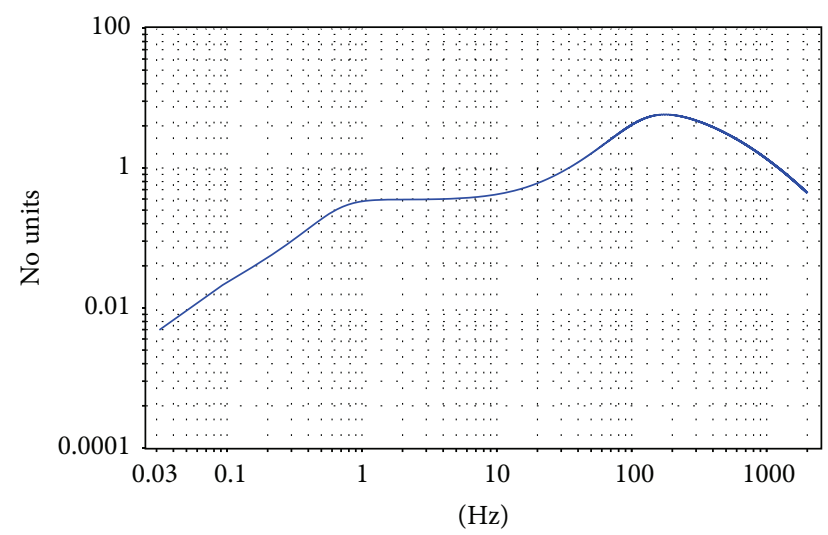

Figure 4: The dimensionless electronic amplifier amplitude response versus frequency.

period. The different sampling rates were tried and the results were compared with the aim of determining an optimal sampling rate when there is no noise transfer from high frequencies to the frequency range of interests as a result of the aliasing.

For each sensor the data processing includes the windowing of the recorded signal, each window 128 seconds in length, calculation of the signal spectrum for each window, and averaging them over all of the windows. The output spectrum has been converted to equivalent angular rate units using the known instrument scale factor $K$. The resulting curves for one of the tested sensors are presented in Figure 5. By comparison of the curves obtained at different sampling rates we can observe that in the $5-100 \mathrm{~Hz}$ frequency range the averaged spectrum at sampling rate 400 sps is significantly higher than the one found at 4000 sps. This effect should be attributed to the aliasing. In other experiments we used only the data obtained at 4000 sps.

The correlation function was calculated for the signals recorded by two METR-11 sensors. The correlation is high (up to 0.9 ) at frequencies corresponding to several peaks at

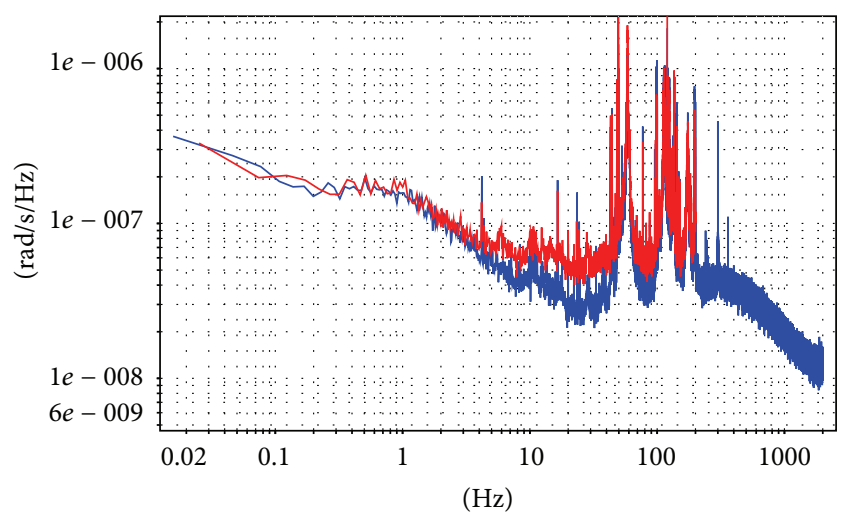

FIgURE 5: Spectra of the METR-11 sensor output signal. The data were recorded at quiet nighttime. Different colors correspond to different sampling rates. Red: 400 sps, blue: 4000 sps.

the spectrum observed in the range $40-200 \mathrm{~Hz}$. These peaks should be associated with the real seismic signal of artificial nature and that reason cannot be considered as a part of the sensors self-noise. Beyond these peaks the correlation is less than 0.2 and we associate the recorded signals with the instrument self-noise. The solid smooth line in Figure 5 is an approximation of the self-noise curve when only parts of the signal corresponding to the frequency ranges with low correlation are used for analysis.

The resulting self-noise frequency behavior as presented by solid smooth line has the following features.

(1) It goes down from $2 \cdot 10^{-7}((\mathrm{rad} / \mathrm{sec}) / \sqrt{\mathrm{Hz}})$ to $6 \cdot$ $10^{-8}((\mathrm{rad} / \mathrm{sec}) / \sqrt{\mathrm{Hz}})$ in the frequency range $1-10 \mathrm{~Hz}$. Here the frequency dependence is $\sim 1 / \sqrt{f}$.

(2) After $\sim 40 \mathrm{~Hz}$ minimum at $\sim 3 \cdot 10^{-8}((\mathrm{rad} / \mathrm{sec}) / \sqrt{\mathrm{Hz}})$ the self-noise increases approximately twice up to 6 . $10^{-8}((\mathrm{rad} / \mathrm{sec}) / \sqrt{\mathrm{Hz}})$ at $200 \mathrm{~Hz}$.

In the next set of the experiments we measured the electronics self-noise. The transducer was replaced by a constant 


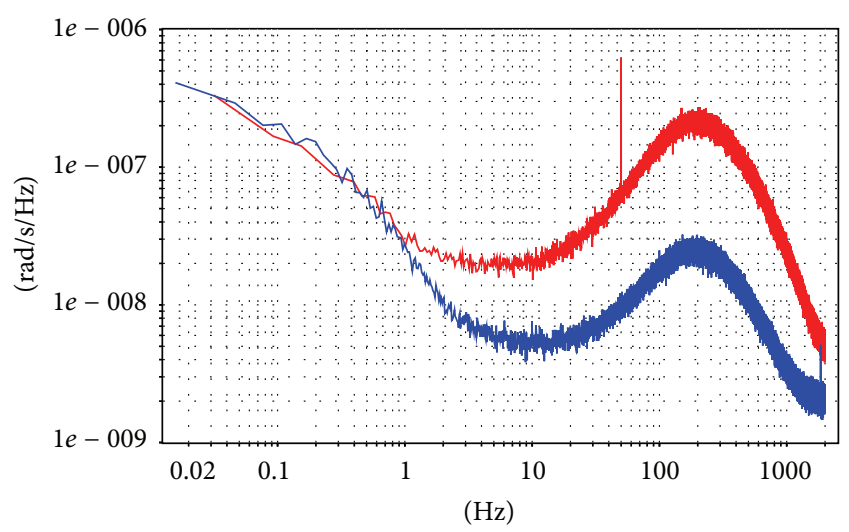

FIGURE 6: Self-noise of the signal conditioning electronics with different input resistors. Red line: $R_{\mathrm{in}}=10 \mathrm{Ohm}$, blue line: $R_{\mathrm{in}}=$ $100 \mathrm{Ohm}$.

resistor modelling its electrical impedance. We used $10 \mathrm{Ohm}$ and $100 \mathrm{Ohm}$ resistors. For convenience of the comparison with the results presented in Figure 5 the electronic noise has been converted from output voltage to the equivalent angular rate. The resulting noise curves are presented in Figure 6. At frequencies close to $1 \mathrm{~Hz}$ the self-noise of the electronics practically does not depend on the resistor value and is $\sim 6$ times lower than the sensor self-noise presented by solid line in Figure 5. At higher frequencies using 10 times smaller resistor means significantly higher (almost 10 times) electronics self-noise. At high frequencies the electronics selfnoise could be either higher or lower than the measured sensor self-noise, depending on the transducer impedance. So, at low frequencies the transducer is the major source of the self-noise, while at higher frequencies the situation depends on the input impedance value and could be opposite when electronic noise achieves and even exceeds the selfnoise produced in the transducer.

2.3. Theoretical Model. Several processes responsible for the MET motion sensors self-noise have been described in the literature $[4,11-13]$. Based on the experimental data presented above we propose a hypothesis that for the frequency range of interest major contributors into the angular sensor self-noise are the convective self-noise investigated in [12] and the selfnoise of the signal conditioning electronics. So, the total noise could be presented by the following equation:

$$
\left\langle U_{f}^{2}\right\rangle_{\text {total }}=\left\langle U_{f}^{2}\right\rangle_{\text {conv }}+\left\langle U_{f}^{2}\right\rangle_{\text {electronics }} .
$$

As observed in the experiments presented at [11], the PSD of the convective noise in the transducer output current at the frequencies above $1 \mathrm{~Hz}$ has approximately $\sim 1 / f$ behavior. Taking into account, according to [10], that in this range the transducer transfer function $W_{\text {trans }}$ is frequency independent we obtain the following formula:

$$
\left\langle U_{f}^{2}\right\rangle_{\text {conv }}=\frac{A}{f}
$$

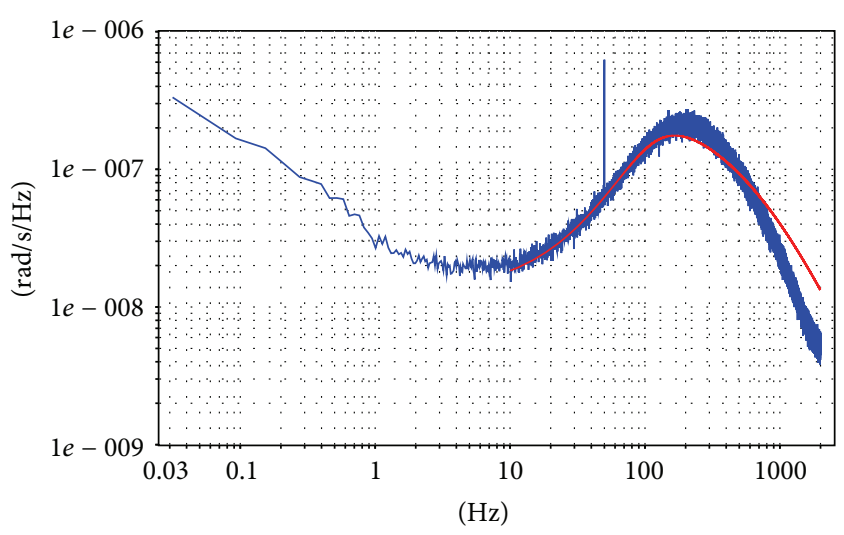

FIGURE 7: Modelling of the electronic board self-noise (blue curve: experiment, red curve: theoretical approximation).

Only little is known about the parameter $A$ dependence on the geometry of the signal converting MET cell and here it is considered as a fitting parameter of a model.

For the electronic self-noise we suppose that most of the noise is generated in the first stage and is the result of a voltage noise of the first stage operational amplifier. After this simplification the electronic self-noise in the instrument operational frequency range could be given by the following:

$$
\left\langle U_{f}^{2}\right\rangle_{\text {electronics }}=\left\langle U_{f}^{2}\right\rangle_{\mathrm{amp}}\left(1+\frac{R_{\mathrm{fb}}}{Z_{\mathrm{in}}}\right)^{2} \frac{\left|W_{2 \mathrm{nd}} W_{3 \mathrm{rd}}\right|^{2}}{K^{2}} .
$$

Here $\left\langle U_{f}^{2}\right\rangle_{\text {amp }}$ is voltage noise power spectral density for the first-stage operational amplifier. $Z_{\text {in }}$ is first-stage input impedance. Values $A$ and $Z_{\text {in }}$ in (5) and (6) are not known a priori and are considered in the following analysis as fitting parameters.

Using (1) and taking into account $\left|W_{1 \mathrm{st}}\right|=R_{\mathrm{fb}}$, formula (6) could be also presented as the following one:

$$
\left\langle U_{f}^{2}\right\rangle_{\text {electromics }}=\left\langle U_{f}^{2}\right\rangle_{\mathrm{amp}}\left(1+\frac{R_{\mathrm{fb}}}{Z_{\mathrm{in}}}\right)^{2} \frac{1}{\left|W_{\text {trans }} R_{\mathrm{fb}}\right|^{2}} .
$$

\section{Results and Discussions}

Let us substitute the following values $Z_{\text {in }}=R_{\text {in }}=$ $40 \mathrm{Ohm} ; R_{\mathrm{fb}}=1 \mathrm{kOhm} ; K=50(\mathrm{~V} /(\mathrm{rad} / \mathrm{sec})) ;\left\langle U_{f}^{2}\right\rangle_{\mathrm{amp}} \approx$ $20 \mathrm{nV} / \sqrt{\mathrm{Hz}}$ [14] in (6). The spectrum of the electronic selfnoise is presented in Figure 7 as a solid black line. The experimental behavior of the electronic noise is in good agreement with the theoretical calculations in the whole range of interest. Some discrepancy is observed at lower frequency, which is, probably, the result of simplifications done when deriving formula (6). Nevertheless, as follows from the experimental data, this difference is not influential since the low frequency electronic noise is only a minor contributor into the total noise of the instrument.

Finally, let us put $A=5.5 \cdot 10^{7}(\mathrm{rad} / \mathrm{sec})^{2}$ in (5) and substitute $\left\langle U_{f}^{2}\right\rangle_{\text {conv }},\left\langle U_{f}^{2}\right\rangle_{\text {electromics }}$ from (5) and (6) into formula (4). The resultant spectrum is presented in Figure 8 as a solid black line. 


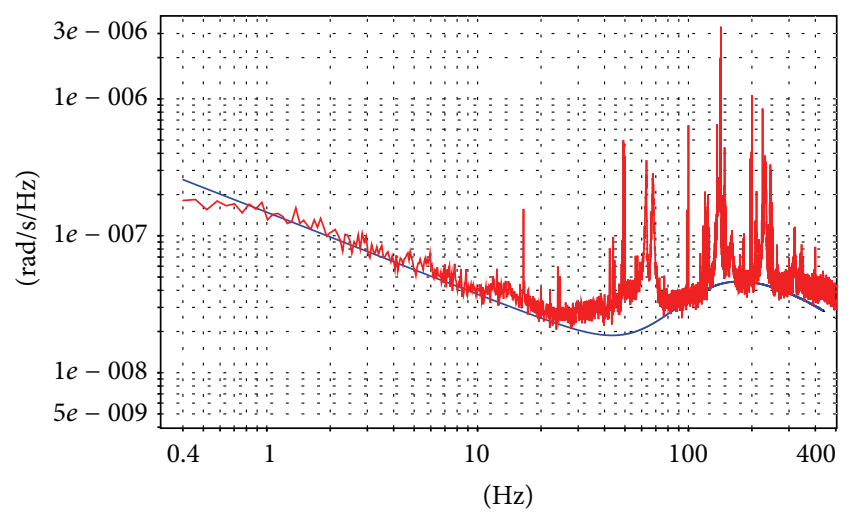

FIGURE 8: Modelling of the angular sensor self-noise (red: electronic self-noise, blue curve: sensors self-noise).

As discussed above the peaks on the experimental curve resulted from the real seismic signals and should not be considered as an instrument self-noise. Taking this into account we can conclude that the theoretical (black line) and the experimental curves (blue line) are in good agreement.

\section{Conclusions}

The model of the MET angular motion sensor self-noise based on the assumption that two physical mechanisms are responsible for the instrument self-noise agrees with experimental data. These two sources are the hydrodynamic convection (first term in (4)) and amplifiers self-noise (second term in (4)). The noise produced by the convection is dominant at low frequencies and then goes down $\sim 1 / f$, while the electronic noise prevails at high frequencies.

The self-noise at low frequencies could be reduced by modification of the transducer. According to earlier analysis [12] this kind of noise could be decreased by using of the sensitive MET cell geometry, characterized by lower Rayleigh number. At high frequencies, as follows from formula (7), the improvement could be achieved by using less noisy operational amplifiers at the first stage. The transducer improvements should be directed toward higher sensitivity $\left|W_{\text {trans }}\right|$ and higher output impedance $\left|Z_{\text {in }}\right|$.

\section{Conflict of Interests}

The authors declare that there is no conflict of interests regarding the publication of this paper.

\section{Acknowledgments}

The results presented in this paper have been obtained under the projects supported by Russian Foundation of Basic Researches (Grant no. 14-07-00232) and Russian Ministry of Education and Science under Project ID RFMEFI57514X0017 and state assignment no. 3.1579.2014/K. Authors sincerely thank Dr. Dmitriy Zaitsev for many fruitful discussions of the topics related to the publication.

\section{References}

[1] L. Chin-Jen and L. George, "Calibration and applications of a rotational sensor," http://www.iris.edu/hq/sits_13_docs/mon/ Lin.pptx.

[2] M. Bernauer, A. Fichtner, and H. Igel, "Measurements of translation, rotation and strain: new approaches to seismic processing and inversion," Journal of Seismology, vol. 16, no. 4, pp. 669-681, 2012.

[3] N. Kapustian, G. Antonovskaya, V. Agafonov, K. Neumoin, and M. Safonov, "Seismic monitoring of linear and rotational oscillations of the multistory buildings in Moscow," Geotechnical, Geological and Earthquake Engineering, vol. 24, pp. 353-363, 2013.

[4] H. Huang, V. Agafonov, and H. Yu, "Molecular electric transducers as motion sensors: a review," Sensors, vol. 13, no. 4, pp. 4581-4597, 2013.

[5] F. Bernauer, J. Wassermann, and H. Igel, "Rotational sensorsa comparison of different sensor types," Journal of Seismology, vol. 16, no. 4, pp. 595-602, 2012.

[6] V. G. Krishtop, V. M. Agafonov, and A. S. Bugaev, “Technological principles of motion parameter transducers based on mass and charge transport in electrochemical microsystems," Russian Journal of Electrochemistry, vol. 48, no. 7, pp. 746-755, 2012.

[7] G. Li, D. Chen, W. He, and J. Wang, "Micro-machined electrochemical seismic sensors with interdigital electrodes," Key Engineering Materials, vol. 503, pp. 61-66, 2012.

[8] W. He, D. Chen, G. Li, and J. Wang, "Low frequency electrochemical accelerometer with low noise based on MEMS," Key Engineering Materials, vol. 503, pp. 75-80, 2012.

[9] H. Huang, B. Carande, R. Tang et al., "A micro seismometer based on molecular electronic transducer technology for planetary exploration," Applied Physics Letters, vol. 102, no. 19, Article ID 193512, 2013.

[10] V. A. Kozlov and M. V. Safonov, "Dynamic characteristic of an electrochemical cell with gauze electrodes in convective diffusion conditions," Russian Journal of Electrochemistry, vol. 40, no. 4, pp. 460-462, 2004.

[11] D. L. Zaitsev, P. V. Dudkin, and V. M. Agafonov, "Fluctuating vortex flows and their contribution to the noise of molecular electronic converters," Izvestiya Vysshikh Uchebnykh Zavedenii, Radiofizika, no. 5, pp. 61-68, 2006 (Russian).

[12] V. M. Agafonov and D. L. Zaitsev, "Convective noise in molecular electronic transducers of diffusion type," Technical Physics, vol. 55, no. 1, pp. 130-136, 2010.

[13] V. A. Kozlov and M. V. Safonov, "Self-noise of molecular electronic transducers," Technical Physics, vol. 48, no. 12, pp. 1579-1582, 2003.

[14] http://www.analog.com/static/imported-files/data_sheets/ AD704.pdf. 

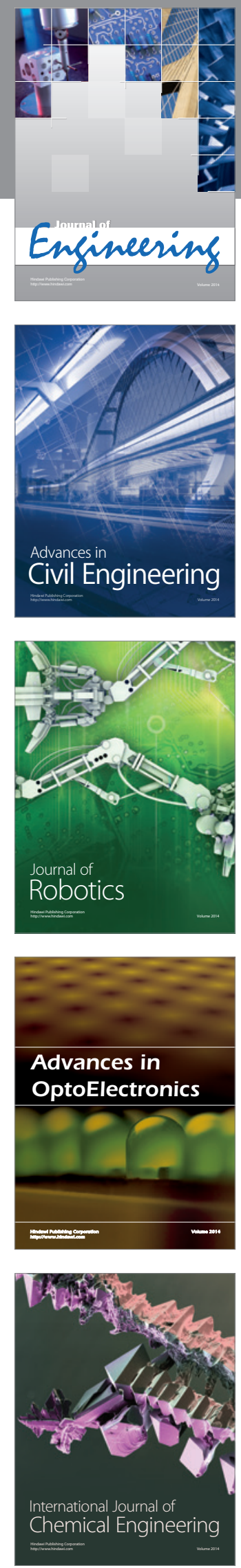

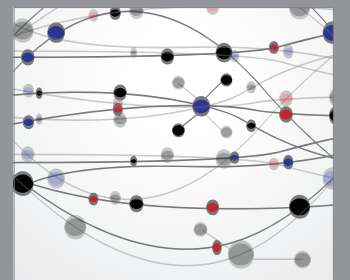

The Scientific World Journal
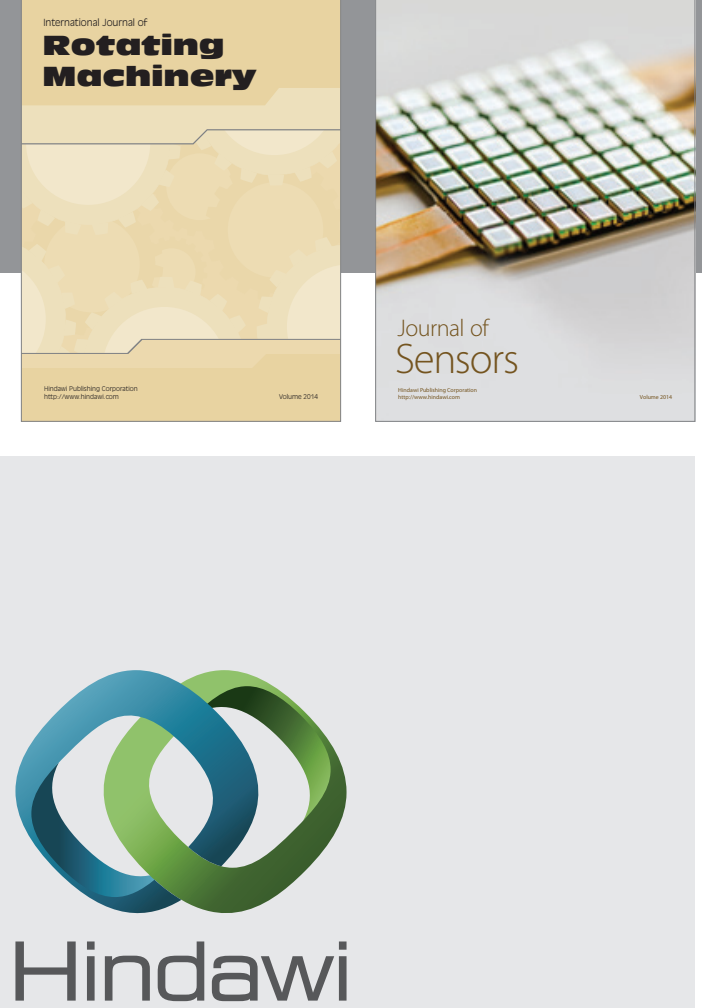

Submit your manuscripts at http://www.hindawi.com
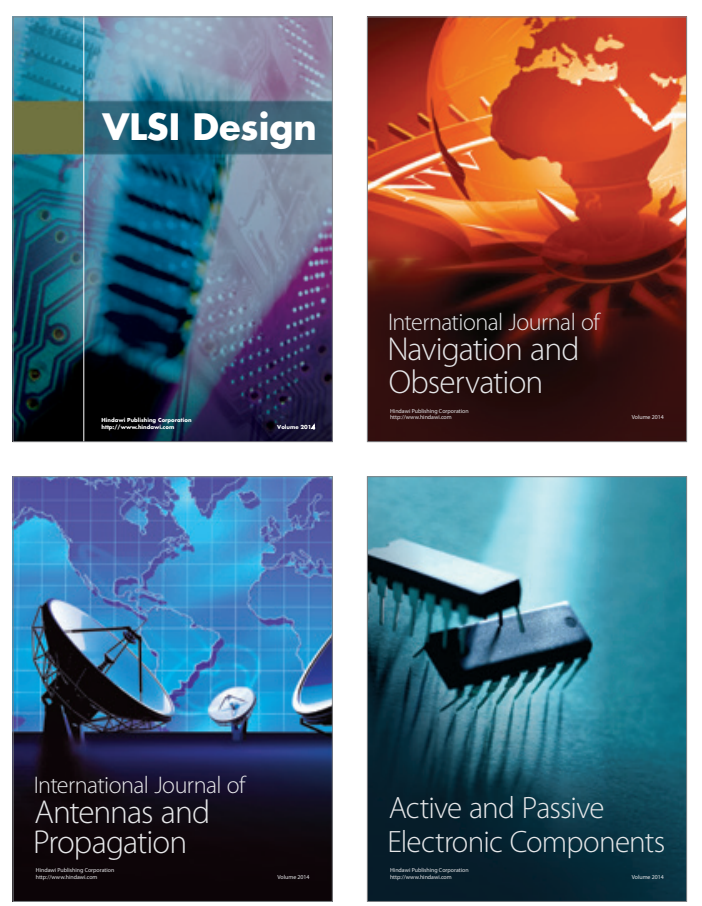
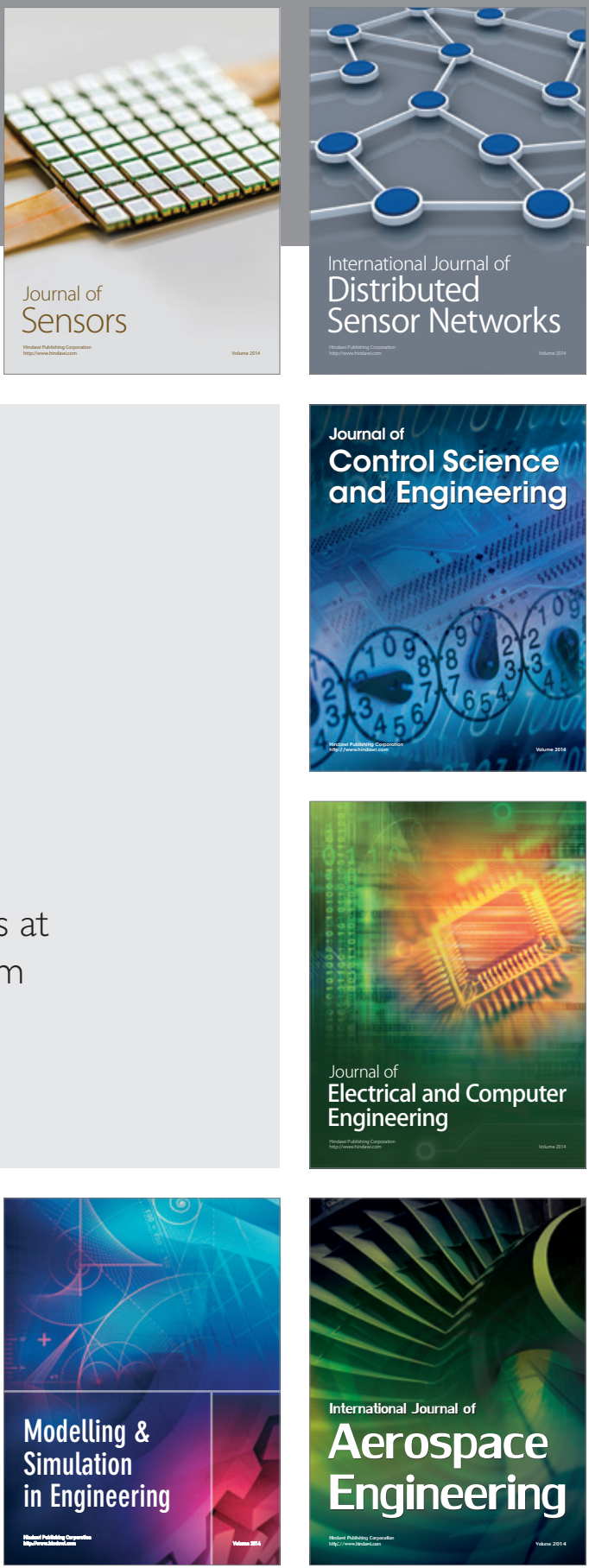

Journal of

Control Science

and Engineering
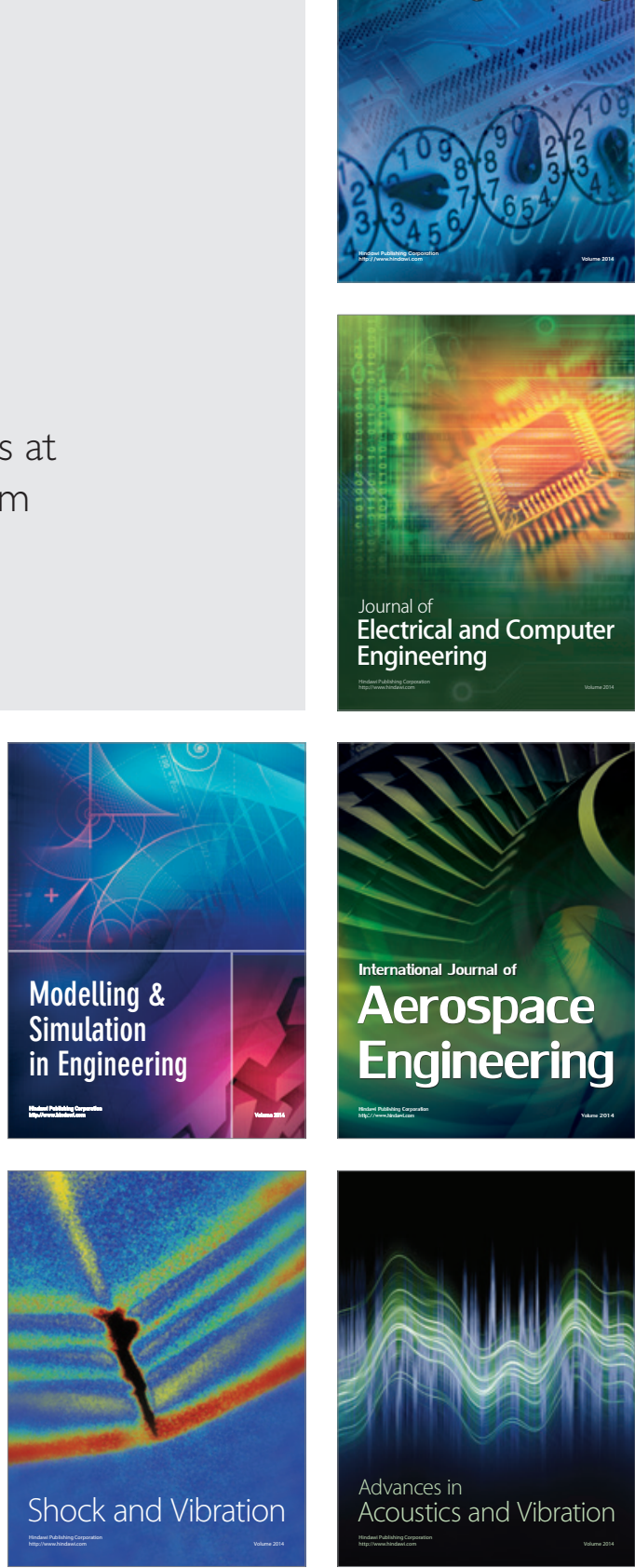